\title{
Well-posedness analysis of a stationary Navier-Stokes hemivariational inequality
}

Min Ling ${ }^{1 *}$ (D) and Weimin Han $^{2}$

\section{${ }^{*}$ Correspondence:}

lingmin@stu.xjtu.edu.cn

${ }^{1}$ School of Mathematics and

Statistics, Xi'an Jiaotong University,

Xi'an, Shaanxi 710049, China

Full list of author information is

available at the end of the article

\section{글 Springer}

\begin{abstract}
This paper provides a well-posedness analysis for a hemivariational inequality of the stationary Navier-Stokes equations by arguments of convex minimization and the Banach fixed point. The hemivariational inequality describes a stationary incompressible fluid flow subject to a nonslip boundary condition and a Clarke subdifferential relation between the total pressure and the normal component of the velocity. Auxiliary Stokes hemivariational inequalities that are useful in proving the solution existence and uniqueness of the Navier-Stokes hemivariational inequality are introduced and analyzed. This treatment naturally leads to a convergent iteration method for solving the Navier-Stokes hemivariational inequality through a sequence of Stokes hemivariational inequalities. Equivalent minimization principles are presented for the auxiliary Stokes hemivariational inequalities which will be useful in developing numerical algorithms.
\end{abstract}

Keywords: Hemivariational inequality; Navier-Stokes equations; Minimization principle; Banach fixed point theorem; Well-posedness; Iteration method; Convergence

\section{Introduction}

Hemivariational inequalities (HVIs) were introduced by Panagiotopoulous in early 1980s, responding to the need of modeling and study of engineering application problems involving non-smooth and non-monotone relations among certain physical quantities. Early research results on hemivariational inequalities are summarized in several books, e.g., $[12,20,21]$. Recent research results on mathematical analysis and applications in contact mechanics of hemivariational inequalities, and more generally variational-hemivariational inequalities, can be found in $[19,22]$. Recent advances on numerical analysis of variationalhemivariational inequalities are summarized in [11].

Hemivariational inequalities also arise for applications in fluid mechanics. A stationary Navier-Stokes HVI is first studied in $[16,17]$, where the solution existence is proved by employing a surjectivity result for a pseudomonotone coercive operator. Then an evolutionary HVI is studied in [18], where the solution existence is proved by applying the Galerkin method to regularized problems, and a solution is obtained as a limit of a sequence of solutions to a regularized problem. In [6], the solution existence is proved for a nonstationary Navier-Stokes HVI through constructing a temporally semi-discrete ap-

(c) The Author(s) 2021. This article is licensed under a Creative Commons Attribution 4.0 International License, which permits use sharing, adaptation, distribution and reproduction in any medium or format, as long as you give appropriate credit to the original author(s) and the source, provide a link to the Creative Commons licence, and indicate if changes were made. The images or other third party material in this article are included in the article's Creative Commons licence, unless indicated otherwise in a credit line to the material. If material is not included in the article's Creative Commons licence and your intended use is not permitted by statutory regulation or exceeds the permitted use, you will need to obtain permission directly from the copyright holder. To view a copy of this licence, visit http://creativecommons.org/licenses/by/4.0/. 
proximation whose solutions converge to a solution of the Navier-Stokes HVI. In [5], well-posedness of a nonstationary Stokes HVI as well as an optimal control problem are studied. Well-posedness of the stationary Stokes HVI is also studied in [14] through an equivalent minimization principle. Recently, several papers have been published on the numerical solution of hemivariational inequalities arising in fluid mechanics. In [4], the mixed finite element method is analyzed for a stationary Stokes HVI with a nonlinear slip boundary condition, and an optimal order error estimate is derived for the mini finite element solution of the Stokes HVI. The nonconforming virtual element method for the same problem is constructed later in [15], where an optimal order error estimate is derived for the lowest-order element solution. A mixed finite element method is studied in [10] for solving a stationary Navier-Stokes HVI with a nonlinear slip boundary condition, and an optimal order error estimate is shown for the mini finite element method.

In this paper, we study the stationary Navier-Stokes HVI considered in $[16,17]$ from a different perspective. We note that the HVI can be used, for example, to model an application where the normal velocity of the fluid is regulated on the boundary to reduce the total pressure. Unlike $[16,17]$ where abstract surjectivity result for pseudomonotone operators is needed, we take an alternative approach using only basic notions and results in functional analysis. We first establish an equivalent minimization principle for an auxiliary problem and prove that there is a unique minimizer of the minimization problem. Under smallness assumptions on the data, we show that the stationary Navier-Stokes HVI has a unique solution by applying the Banach fixed point argument. Our approach naturally leads to a convergent iteration algorithm so that the solution of the stationary NavierStokes HVI can be approximated by a sequence of solutions of the simpler Stokes HVIs. We also provide a Lipschitz continuous dependence result for the solution of the stationary Navier-Stokes HVI on the right-hand side function.

Let us recall the stationary Navier-Stokes HVI. Let $\Omega \subset \mathbb{R}^{d}(d=2,3)$ be a bounded simply connected set with a smooth boundary $\Gamma=\partial \Omega$. Denote by $\boldsymbol{n}$ the unit outward normal to $\Gamma$. For a vector-valued function $\boldsymbol{u}$ on the boundary, let $u_{n}=\boldsymbol{u} \cdot \boldsymbol{n}$ and $\boldsymbol{u}_{\tau}=\boldsymbol{u}-u_{n} \boldsymbol{n}$ be the normal component and the tangential component, respectively. The standard form of the Navier-Stokes equations is

$$
-v \Delta \boldsymbol{u}+(\boldsymbol{u} \cdot \nabla) \boldsymbol{u}+\nabla p=\boldsymbol{f}, \quad \operatorname{div} \boldsymbol{u}=0 \quad \text { in } \Omega,
$$

where $v$ and $\boldsymbol{f}$ are given, $v>0$ being the kinematic viscosity of the fluid and $\boldsymbol{f}$ the density of volume forces. The unknown variables are the fluid velocity field $\boldsymbol{u}=\left(u_{1}, \ldots, u_{d}\right)^{T}$ and the pressure $p$. The incompressibility property is reflected in the divergence free condition $\operatorname{div} \boldsymbol{u}=0$. In relation to the boundary condition considered in this paper, we use the identities (cf. [7])

$$
\begin{aligned}
& (\boldsymbol{u} \cdot \nabla) \boldsymbol{u}=\operatorname{curl} \boldsymbol{u} \times \boldsymbol{u}+\frac{1}{2} \nabla|\boldsymbol{u}|^{2}, \\
& -\Delta \boldsymbol{u}=\operatorname{curl} \operatorname{curl} \boldsymbol{u}-\nabla \operatorname{div} \boldsymbol{u}
\end{aligned}
$$

and rewrite the Navier-Stokes equations with the Lamb formulation [13] as

$$
v \operatorname{curl} \operatorname{curl} \boldsymbol{u}+\operatorname{curl} \boldsymbol{u} \times \boldsymbol{u}+\nabla \widetilde{p}=\boldsymbol{f} \quad \text { in } \Omega,
$$


$\operatorname{div} \boldsymbol{u}=0 \quad$ in $\Omega$.

Here, curl is the curl operator, and $\widetilde{p}=p+|\boldsymbol{u}|^{2} / 2$ is the total pressure. For $d=3$, the curl operator is defined by the formula

$$
\operatorname{curl} \boldsymbol{u}=\left(\frac{\partial u_{3}}{\partial x_{2}}-\frac{\partial u_{2}}{\partial x_{3}}, \frac{\partial u_{1}}{\partial x_{3}}-\frac{\partial u_{3}}{\partial x_{1}}, \frac{\partial u_{2}}{\partial x_{1}}-\frac{\partial u_{1}}{\partial x_{2}}\right)^{T} .
$$

The curl operator for a two-dimensional vector can be converted to that for a threedimensional vector through the following procedure: we extend vector $\boldsymbol{u}\left(x_{1}, x_{2}\right)=$ $\left(u_{1}\left(x_{1}, x_{2}\right), u_{2}\left(x_{1}, x_{2}\right)\right)^{T}$ by $\boldsymbol{u}\left(x_{1}, x_{2}, x_{3}\right)=\left(u_{1}\left(x_{1}, x_{2}\right), u_{2}\left(x_{1}, x_{2}\right), 0\right)^{T}$. Then,

$$
\operatorname{curl} \boldsymbol{u}=\left(0,0, \frac{\partial u_{2}}{\partial x_{1}}-\frac{\partial u_{1}}{\partial x_{2}}\right)^{T} .
$$

The Navier-Stokes equations (1.2)-(1.3) are supplemented with the following boundary conditions:

$$
\mathbf{u}_{\tau}=\mathbf{0}, \quad \tilde{p} \in \partial \psi\left(u_{n}\right) \quad \text { on } \Gamma .
$$

Here the super-potential $\psi: \Gamma \times \mathbb{R} \rightarrow \mathbb{R}$ is locally Lipschitz continuous with respect to its second argument. To simplify the notation, we write $\psi\left(u_{n}\right)$ for $\psi\left(\boldsymbol{x}, u_{n}\right)$, and $\partial \psi$ is the subdifferential of $\psi$ in the sense of Clarke with respect to its second argument. The first part of condition (1.4), i.e., $\boldsymbol{u}_{\tau}=\mathbf{0}$, is known as a nonslip boundary condition. The second part is to regulate the normal velocity $u_{n}$ to reduce the total pressure $\widetilde{p}$ on $\Gamma$.

The rest of the paper is organized as follows. In Sect. 2, we review the notions of the generalized directional derivative and the subdifferential in the sense of Clarke, and some of their basic properties. We also introduce the Navier-Stokes hemivariational inequality formulation for problem (1.2)-(1.4). In Sect. 3, we show the well-posedness of the Navier-Stokes hemivariational inequality. To prove the solution existence and uniqueness of the Navier-Stokes HVI, we adopt arguments of the minimization principle for auxiliary Stokes hemivariational inequalities and apply the Banach fixed point theorem. This naturally leads to a convergent iteration algorithm for the Navier-Stokes HVI through solving Stokes hemivariational inequalities. We further show the Lipschitz continuous dependence of the solution of the Navier-Stokes HVI on the right-hand side.

\section{Navier-Stokes hemivariational inequality}

In this section, we present the hemivariational inequality formulation for problem (1.2)(1.4). We first recall the definitions of the generalized directional derivative and generalized subdifferential in the sense of Clarke for a locally Lipschitz function and review some basic properties.

Definition 2.1 Let $V$ be a Banach space and denote by $V^{*}$ its dual. Let $\Psi: V \rightarrow \mathbb{R}$ be a locally Lipschitz functional. The generalized (Clarke) directional derivative of $\Psi$ at $u \in V$ in the direction $v \in V$ is defined by

$$
\Psi^{0}(u ; v)=\limsup _{w \rightarrow u, \lambda \downarrow 0} \frac{\Psi(w+\lambda v)-\Psi(w)}{\lambda} .
$$


The generalized gradient (subdifferential) of $\Psi$ at $u$ is defined by

$$
\partial \Psi(u)=\left\{\zeta \in V^{*}: \Psi^{0}(u ; v) \geq\langle\zeta, v\rangle \forall v \in V\right\} .
$$

Given the generalized subdifferential, we can compute the generalized directional derivative through the formula [2, Proposition 2.1.2]

$$
\Psi^{0}(u ; v)=\max \{\langle\xi, v\rangle: \xi \in \partial \Psi(u)\} \quad \forall u, v \in V .
$$

If $\Psi_{1}, \Psi_{2}: V \rightarrow \mathbb{R}$ are locally Lipschitz continuous, then [2, Proposition 2.3.3]

$$
\partial\left(\Psi_{1}+\Psi_{2}\right)(u) \subset \partial \Psi_{1}(u)+\partial \Psi_{2}(u) \quad \forall u \in V,
$$

or equivalently,

$$
\left(\Psi_{1}+\Psi_{2}\right)^{0}(u ; v) \leq \Psi_{1}^{0}(u ; v)+\Psi_{2}^{0}(u ; v) \quad \forall u, v \in V .
$$

In the analysis of the Navier-Stokes hemivariational inequality, we will need the following result [3, Theorem 3.4].

Lemma 2.2 Let $V$ be a real Banach space, and let $g: V \rightarrow \mathbb{R}$ be locally Lipschitz continuous. Then $g$ is strongly convex on $V$ with a constant $\alpha>0$, i.e.,

$$
g(\lambda u+(1-\lambda) v) \leq \lambda g(u)+(1-\lambda) g(v)-\alpha \lambda(1-\lambda)\|u-v\|_{V}^{2} \quad \forall u, v \in V, \forall \lambda \in[0,1],
$$

if and only if $\partial g$ is strongly monotone on $V$ with a constant $2 \alpha$, i.e.,

$$
\langle\xi-\eta, u-v\rangle \geq 2 \alpha\|u-v\|_{V}^{2} \quad \forall u, v \in V, \xi \in \partial g(u), \eta \in \partial g(v)
$$

A proof of the next result can be found in [8, Proposition 2.5].

Proposition 2.3 Let $V$ be a real Hilbert space, and let $g: V \rightarrow \mathbb{R}$ be a locally Lipschitz continuous and strongly convex functional on $V$ with a constant $\alpha>0$. Then there exist two constants $\bar{c}_{0}$ and $\bar{c}_{1}$ such that

$$
g(v) \geq \alpha\|v\|_{V}^{2}+\bar{c}_{0}+\bar{c}_{1}\|v\|_{V} \quad \forall v \in V .
$$

Consequently, $g(\cdot)$ is coercive on $V$.

We will make use of the function space

$$
\boldsymbol{V}=\left\{\boldsymbol{v} \in \boldsymbol{H}^{1}(\Omega): \operatorname{div} \boldsymbol{v}=0 \text { in } \Omega, \boldsymbol{v}_{\tau}=\mathbf{0} \text { on } \Gamma\right\}
$$

The following inequality holds [7, Proposition 3.1]:

$$
\|\boldsymbol{v}\|_{1, \Omega} \leq c\|\operatorname{curl} \boldsymbol{v}\|_{0, \Omega} \quad \forall \boldsymbol{v} \in \boldsymbol{V}
$$


where the constant $c>0$ depends on $\Omega$ and $\Gamma$. Thus $\|\operatorname{curl} \cdot\|_{0, \Omega}$ defines a norm and is equivalent to the standard $\boldsymbol{H}^{1}(\Omega)$-norm on $\boldsymbol{V}$. We use $\|\cdot\|_{\boldsymbol{V}}=\|\operatorname{curl} \cdot\|_{0, \Omega}$ for the norm on $\boldsymbol{V}$. By the Sobolev trace theorem, we have the inequality

$$
\left\|v_{n}\right\|_{0, \Gamma} \leq \lambda_{0}^{-1 / 2}\|\boldsymbol{v}\|_{\boldsymbol{v}} \quad \forall \boldsymbol{v} \in \boldsymbol{V}
$$

where $\lambda_{0}>0$ is the smallest eigenvalue of the eigenvalue problem

$$
\mathbf{u} \in \boldsymbol{V}, \quad \int_{\Omega} \operatorname{curl} \boldsymbol{u} \cdot \operatorname{curl} \mathbf{v} d x=\lambda \int_{\Gamma} u_{n} v_{n} d s \quad \forall \mathbf{v} \in \mathbf{V} .
$$

Define

$$
\begin{aligned}
& a(\boldsymbol{u}, \boldsymbol{v})=v \int_{\Omega} \operatorname{curl} \boldsymbol{u} \cdot \operatorname{curl} \boldsymbol{v} d x \quad \forall \mathbf{u}, \boldsymbol{v} \in \boldsymbol{V} \\
& b(\boldsymbol{u}, \boldsymbol{v}, \boldsymbol{w})=\int_{\Omega}(\operatorname{curl} \boldsymbol{u} \times \boldsymbol{v}) \cdot \boldsymbol{w} d x \quad \forall \boldsymbol{u}, \boldsymbol{v}, \boldsymbol{w} \in \boldsymbol{V}, \\
& \langle\boldsymbol{f}, \boldsymbol{v}\rangle=\int_{\Omega} \boldsymbol{f} \cdot \boldsymbol{v} d x \quad \forall \boldsymbol{v} \in \boldsymbol{V} .
\end{aligned}
$$

Then the following hemivariational inequality formulation can be derived for problem (1.2)-(1.4) [17].

Problem 2.4 Find $\boldsymbol{u} \in \boldsymbol{V}$ such that

$$
a(\boldsymbol{u}, \boldsymbol{v})+b(\boldsymbol{u}, \boldsymbol{u}, \boldsymbol{v})+\int_{\Gamma} \psi^{0}\left(u_{n} ; v_{n}\right) d s \geq\langle\boldsymbol{f}, \boldsymbol{v}\rangle \quad \forall \mathbf{v} \in \boldsymbol{V} .
$$

We note that the bilinear form $a(\cdot, \cdot)$ is continuous and coercive on $\boldsymbol{V}$ :

$$
a(\boldsymbol{u}, \boldsymbol{v}) \leq v\|\boldsymbol{u}\|_{\boldsymbol{v}}\|\boldsymbol{v}\|_{\boldsymbol{v}} \quad \text { and } \quad a(\boldsymbol{v}, \boldsymbol{v})=v\|\boldsymbol{v}\|_{\boldsymbol{v}}^{2} \quad \forall \boldsymbol{u}, \boldsymbol{v} \in \boldsymbol{V} .
$$

The trilinear form $b(\cdot, \cdot, \cdot)$ is continuous on $\boldsymbol{V}$ :

$$
b(\boldsymbol{u}, \boldsymbol{v}, \boldsymbol{w}) \leq c_{b}\|\boldsymbol{u}\|_{\boldsymbol{v}}\|\boldsymbol{v}\|_{\boldsymbol{v}}\|\boldsymbol{w}\|_{\boldsymbol{v}} \quad \forall \boldsymbol{u}, \boldsymbol{v}, \boldsymbol{w} \in \boldsymbol{V},
$$

and satisfies

$$
b(\boldsymbol{u}, \boldsymbol{v}, \boldsymbol{w})=-b(\boldsymbol{u}, \boldsymbol{w}, \boldsymbol{v}) \quad \text { and } \quad b(\boldsymbol{u}, \boldsymbol{v}, \boldsymbol{v})=0 \quad \forall \boldsymbol{u}, \boldsymbol{v}, \boldsymbol{w} \in \boldsymbol{V} .
$$

Concerning the superpotential $\psi$, we assume the following properties:

$H(\psi) . \psi: \Gamma \times \mathbb{R} \rightarrow \mathbb{R}$ is such that

(i) $\psi(\cdot, \xi)$ is measurable on $\Gamma$ for all $\xi \in \mathbb{R}$ and $\psi(\cdot, 0) \in L^{1}(\Gamma)$;

(ii) $\psi(\boldsymbol{x}, \cdot)$ is locally Lipschitz on $\mathbb{R}$ for a.e. $\boldsymbol{x} \in \Gamma$;

(iii) $|\eta| \leq c_{0}+c_{1}|\xi| \forall \xi \in \mathbb{R}, \eta \in \partial \psi(\boldsymbol{x}, \xi)$ a.e. $\boldsymbol{x} \in \Gamma$ with $c_{0}, c_{1} \geq 0$;

(iv) $\psi^{0}\left(\boldsymbol{x}, \xi_{1} ; \xi_{2}-\xi_{1}\right)+\psi^{0}\left(\boldsymbol{x}, \xi_{2} ; \xi_{1}-\xi_{2}\right) \leq m\left|\xi_{1}-\xi_{2}\right|^{2} \forall \xi_{1}, \xi_{2} \in \mathbb{R}$ a.e. $\boldsymbol{x} \in \Gamma$ with $m \geq 0$.

By $(2.1), H(\psi)$ (iii) implies

$$
\left|\psi^{0}\left(\xi_{1} ; \xi_{2}\right)\right| \leq\left(c_{0}+c_{1}\left|\xi_{1}\right|\right)\left|\xi_{2}\right| \quad \forall \xi_{1}, \xi_{2} \in \mathbb{R} .
$$


Define a functional $\Psi: \boldsymbol{V} \rightarrow \mathbb{R}$ by

$$
\Psi(\boldsymbol{v})=\int_{\Gamma} \psi\left(v_{n}\right) d s \quad \forall \boldsymbol{v} \in \boldsymbol{V}
$$

Then, by [17, Lemma 13], under assumption $H(\psi), \Psi$ is well-defined and locally Lipschitz continuous on $\boldsymbol{V}$, and

$$
\Psi^{0}(\boldsymbol{u} ; \boldsymbol{v}) \leq \int_{\Gamma} \psi^{0}\left(u_{n} ; v_{n}\right) d s \quad \forall \boldsymbol{u}, \boldsymbol{v} \in \boldsymbol{V}
$$

Combining $H(\psi)$ (iv), (2.5), and (2.9), we have, for $\boldsymbol{v}_{1}, \boldsymbol{v}_{2} \in \boldsymbol{V}$,

$$
\begin{aligned}
\Psi^{0}\left(\boldsymbol{v}_{1} ; \boldsymbol{v}_{2}-\boldsymbol{v}_{1}\right)+\Psi^{0}\left(\boldsymbol{v}_{2} ; \boldsymbol{v}_{1}-\boldsymbol{v}_{2}\right) & \leq \int_{\Gamma}\left[\psi^{0}\left(v_{1 n} ; v_{2 n}-v_{1 n}\right)+\psi^{0}\left(v_{2 n} ; v_{1 n}-v_{2 n}\right)\right] d s \\
& \leq \int_{\Gamma} m\left|v_{1 n}-v_{2 n}\right|^{2} d s \leq m \lambda_{0}^{-1}\left\|\boldsymbol{v}_{1}-\boldsymbol{v}_{2}\right\|_{\boldsymbol{V}}^{2}
\end{aligned}
$$

It is known that (2.10) is equivalent to [19]

$$
\left\langle\boldsymbol{\eta}_{1}-\boldsymbol{\eta}_{2}, \boldsymbol{v}_{1}-\boldsymbol{v}_{2}\right\rangle \geq-m \lambda_{0}^{-1}\left\|\boldsymbol{v}_{1}-\boldsymbol{v}_{2}\right\|_{\boldsymbol{V}}^{2} \quad \forall \boldsymbol{v}_{i} \in \boldsymbol{V}, \boldsymbol{\eta}_{i} \in \partial \Psi\left(\boldsymbol{v}_{i}\right), i=1,2
$$

\section{Well-posedness analysis}

This section is devoted to a study of the well-posedness of Problem 2.4.

\subsection{Study of auxiliary problems}

We first introduce and study an auxiliary problem. Let $\boldsymbol{w} \in \boldsymbol{V}$ be given.

Problem 3.1 Find $\boldsymbol{u} \in \boldsymbol{V}$ such that

$$
a(\boldsymbol{u}, \boldsymbol{v})+\Psi^{0}(\boldsymbol{u} ; \boldsymbol{v}) \geq\langle\boldsymbol{f}, \boldsymbol{v}\rangle-b(\boldsymbol{w}, \boldsymbol{w}, \boldsymbol{v}) \quad \forall \boldsymbol{v} \in \boldsymbol{V}
$$

We adopt the idea presented in $[8,9]$ and study the solution existence of Problem 3.1 through an equivalent minimization problem. Define a linear operator $A: \boldsymbol{V} \rightarrow \boldsymbol{V}^{*}$ by

$$
\langle A \boldsymbol{u}, \boldsymbol{v}\rangle=a(\boldsymbol{u}, \boldsymbol{v}) \quad \forall \boldsymbol{u}, \boldsymbol{v} \in \boldsymbol{V}
$$

Then $A \in \mathcal{L}\left(\boldsymbol{V}, \boldsymbol{V}^{*}\right)$. Define a functional $\boldsymbol{I} \in \boldsymbol{V}^{*}$ by

$$
\langle\boldsymbol{I}, \boldsymbol{v}\rangle=\langle\boldsymbol{f}, \boldsymbol{v}\rangle-b(\boldsymbol{w}, \boldsymbol{w}, \boldsymbol{v}) \quad \forall \boldsymbol{v} \in \boldsymbol{V} .
$$

We introduce an energy functional

$$
E(\boldsymbol{v})=\frac{1}{2} a(\boldsymbol{v}, \boldsymbol{v})+\Psi(\boldsymbol{v})-\langle\boldsymbol{I}, \boldsymbol{v}\rangle \quad \forall \boldsymbol{v} \in \boldsymbol{V}
$$

and consider a corresponding minimization problem:

Problem 3.2 Find $\boldsymbol{u} \in \boldsymbol{V}$ such that

$$
E(\boldsymbol{u})=\inf \{E(\boldsymbol{v}): \boldsymbol{v} \in \boldsymbol{V}\}
$$


The next result shows that Problem 3.1 and Problem 3.2 are equivalent.

Theorem 3.3 Assume $H(\psi), \boldsymbol{f} \in \boldsymbol{V}^{*}$, and

$$
m<\nu \lambda_{0}
$$

Then, for any $\boldsymbol{w} \in \boldsymbol{V}$, Problem 3.1 and Problem 3.2 have the same unique solution $\mathbf{u} \in \boldsymbol{V}$.

Proof Obviously, $E: \boldsymbol{V} \rightarrow \mathbb{R}$ is locally Lipschitz continuous. By (2.2),

$$
\partial E(\boldsymbol{v}) \subset A \boldsymbol{v}+\partial \Psi(\boldsymbol{v})-\boldsymbol{I} .
$$

For $i=1,2$, with $\boldsymbol{v}_{i} \in \boldsymbol{V}$ and $\zeta_{i} \in \partial E\left(\boldsymbol{v}_{i}\right)$, we have

$$
\zeta_{i}=A \boldsymbol{v}_{i}+\boldsymbol{\eta}_{i}-\boldsymbol{l}, \quad \boldsymbol{\eta}_{i} \in \partial \Psi\left(\boldsymbol{v}_{i}\right)
$$

By (2.11),

$$
\left\langle\zeta_{1}-\zeta_{2}, \boldsymbol{v}_{1}-\boldsymbol{v}_{2}\right\rangle=a\left(\boldsymbol{v}_{1}-\boldsymbol{v}_{2}, \boldsymbol{v}_{1}-\boldsymbol{v}_{2}\right)+\left\langle\boldsymbol{\eta}_{1}-\boldsymbol{\eta}_{2}, \boldsymbol{v}_{1}-\boldsymbol{v}_{2}\right\rangle \geq\left(\nu-m \lambda_{0}^{-1}\right)\left\|\boldsymbol{v}_{1}-\boldsymbol{v}_{2}\right\|_{\boldsymbol{v}}^{2}
$$

Since $m<\nu \lambda_{0}$, by Lemma 2.2, the energy functional $E$ is strongly convex on $\boldsymbol{V}$. Moreover, by Proposition 2.3, $E$ is coercive on $\boldsymbol{V}$. Then the minimization Problem 3.2 has a unique solution $\boldsymbol{u} \in \boldsymbol{V}$ [1, Sect. 3.3.2], which satisfies the relation

$$
E^{0}(\boldsymbol{u} ; \boldsymbol{v}) \geq 0 \quad \forall \boldsymbol{v} \in \boldsymbol{V} .
$$

By (2.3),

$$
E^{0}(\boldsymbol{u} ; \boldsymbol{v}) \leq a(\boldsymbol{u}, \boldsymbol{v})+\Psi^{0}(\boldsymbol{u} ; \boldsymbol{v})-\langle\boldsymbol{l}, \boldsymbol{v}\rangle
$$

Thus,

$$
a(\boldsymbol{u}, \boldsymbol{v})+\Psi^{0}(\boldsymbol{u} ; \boldsymbol{v}) \geq\langle\boldsymbol{I}, \boldsymbol{v}\rangle \quad \forall \boldsymbol{v} \in \boldsymbol{V}
$$

and $\boldsymbol{u} \in \boldsymbol{V}$ is a solution of Problem 3.1.

Let us prove the solution uniqueness for Problem 3.1. Suppose that $\tilde{\boldsymbol{u}} \in \boldsymbol{V}$ is another solution of Problem 3.1. Then

$$
a(\tilde{\boldsymbol{u}}, \boldsymbol{v})+\Psi^{0}(\tilde{\boldsymbol{u}} ; \boldsymbol{v}) \geq\langle\boldsymbol{I}, \boldsymbol{v}\rangle \quad \forall \boldsymbol{v} \in \boldsymbol{V} .
$$

We take $\boldsymbol{v}=\tilde{\boldsymbol{u}}-\boldsymbol{u}$ in (3.1), take $\boldsymbol{v}=\boldsymbol{u}-\tilde{\boldsymbol{u}}$ in (3.6), and add the two inequalities to obtain

$$
a(\tilde{\boldsymbol{u}}-\boldsymbol{u}, \tilde{\boldsymbol{u}}-\boldsymbol{u}) \leq \Psi^{0}(\tilde{\boldsymbol{u}} ; \boldsymbol{u}-\tilde{\boldsymbol{u}})+\Psi^{0}(\boldsymbol{u} ; \tilde{\boldsymbol{u}}-\boldsymbol{u}) .
$$

By (2.10),

$$
v\|\tilde{\boldsymbol{u}}-\boldsymbol{u}\|_{\boldsymbol{v}}^{2} \leq m \lambda_{0}^{-1}\|\tilde{\boldsymbol{u}}-\boldsymbol{u}\|_{\boldsymbol{v}}^{2}
$$


By the smallness condition (3.4), we conclude that $\tilde{\boldsymbol{u}}=\boldsymbol{u}$, i.e., a solution of Problem 3.1. Therefore, $\boldsymbol{u} \in \boldsymbol{V}$ is a solution of Problem 3.1 if and only if it is a solution of Problem 3.2, and both problems admit a unique solution.

Then, we consider a variant of Problem 3.1.

Problem 3.4 Find $\boldsymbol{u} \in \boldsymbol{V}$ such that

$$
a(\boldsymbol{u}, \boldsymbol{v})+\int_{\Gamma} \psi^{0}\left(u_{n} ; v_{n}\right) d s \geq\langle\boldsymbol{f}, \boldsymbol{v}\rangle-b(\boldsymbol{w}, \boldsymbol{w}, \boldsymbol{v}) \quad \forall \boldsymbol{v} \in \boldsymbol{V}
$$

Theorem 3.5 Assume $H(\psi), \boldsymbol{f} \in \boldsymbol{V}^{*}$, and (3.4). Then, for any $\boldsymbol{w} \in \boldsymbol{V}$, Problem 3.4 has a unique solution $\mathbf{u} \in \boldsymbol{V}$, which is also the unique solution of Problem 3.1 and Problem 3.2.

Proof From Theorem 3.3, we know that under the stated assumptions, Problem 3.1 has a unique solution $\boldsymbol{u} \in \boldsymbol{V}$, which is the unique solution of Problem 3.2. Since

$$
\Psi^{0}(\boldsymbol{u} ; \boldsymbol{v}) \leq \int_{\Gamma} \psi^{0}\left(u_{n} ; v_{n}\right) d s
$$

we see that the solution $\boldsymbol{u}$ satisfies inequality (3.7). In other words, $\boldsymbol{u} \in \boldsymbol{V}$ is also a solution of Problem 3.4.

The uniqueness of a solution to Problem 3.4 can be proved similarly as in the proof of Theorem 3.3, and is hence omitted. Thus, the statement of Theorem 3.5 is valid.

\subsection{Solution existence and uniqueness}

In this subsection, we show the solution existence and uniqueness for Problem 2.4 based on the result from previous subsection, through a Banach fixed point argument.

For given $\boldsymbol{w} \in \boldsymbol{V}$, let us first bound $\|\boldsymbol{u}\|_{\boldsymbol{v}}$ for the solution of Problem 3.4. We take $\boldsymbol{v}=-\boldsymbol{u}$ in (3.7) to obtain

$$
a(\boldsymbol{u}, \boldsymbol{u}) \leq \int_{\Gamma} \psi^{0}\left(u_{n} ;-u_{n}\right) d s+\langle\boldsymbol{f}, \boldsymbol{u}\rangle-b(\boldsymbol{w}, \boldsymbol{w}, \boldsymbol{u}) .
$$

By $H(\psi)($ iv) and (2.8),

$$
\begin{aligned}
\int_{\Gamma} \psi^{0}\left(u_{n} ;-u_{n}\right) d s & \leq \int_{\Gamma} m\left|u_{n}\right|^{2} d s-\int_{\Gamma} \psi^{0}\left(0 ; u_{n}\right) d s \\
& \leq \int_{\Gamma} m\left|u_{n}\right|^{2} d s+\int_{\Gamma} c_{0}\left|u_{n}\right| d s \\
& \leq m \lambda_{0}^{-1}\|\boldsymbol{u}\|_{\boldsymbol{V}}^{2}+c_{0} \lambda_{0}^{-1 / 2}|\Gamma|^{1 / 2}\|\boldsymbol{u}\| \boldsymbol{v} .
\end{aligned}
$$

We derive from (3.8) that

$$
\left(v-m \lambda_{0}^{-1}\right)\|\boldsymbol{u}\|_{\boldsymbol{v}}^{2} \leq c_{0} \lambda_{0}^{-1 / 2}|\Gamma|^{1 / 2}\|\boldsymbol{u}\|_{\boldsymbol{v}}+\|\boldsymbol{f}\| \boldsymbol{V}^{*}\|\boldsymbol{u}\| \boldsymbol{v}+c_{b}\|\boldsymbol{w}\|_{\boldsymbol{V}}^{2}\|\boldsymbol{u}\|_{\boldsymbol{v}}
$$

Hence,

$$
\|\boldsymbol{u}\|_{\boldsymbol{v}} \leq \frac{c_{0} \lambda_{0}^{-1 / 2}|\Gamma|^{1 / 2}+\|\boldsymbol{f}\|_{\boldsymbol{v}^{*}}}{v-m \lambda_{0}^{-1}}+\frac{c_{b}\|\boldsymbol{w}\|_{\boldsymbol{v}}^{2}}{v-m \lambda_{0}^{-1}}
$$


Introduce a bounded set of $\boldsymbol{V}$ by

$$
\boldsymbol{K}_{\boldsymbol{f}}=\left\{\boldsymbol{v} \in \boldsymbol{V}:\|\boldsymbol{v}\|_{\boldsymbol{v}} \leq \alpha_{\boldsymbol{f}}\right\},
$$

where

$$
\alpha_{\boldsymbol{f}}=\frac{2\left(c_{0} \lambda_{0}^{-1 / 2}|\Gamma|^{1 / 2}+\|\boldsymbol{f}\| \boldsymbol{v}^{*}\right)}{v-m \lambda_{0}^{-1}} .
$$

Proposition 3.6 Assume $H(\psi), \boldsymbol{f} \in \boldsymbol{V}^{*}$, and the smallness condition

$$
0<2 c_{b} \alpha_{\boldsymbol{f}}<v-m \lambda_{0}^{-1} .
$$

Then, for any $\boldsymbol{w} \in \boldsymbol{K}_{\boldsymbol{f}}$, the solution $\boldsymbol{u}$ of Problem 3.4 also belongs to the set $\boldsymbol{K}_{\boldsymbol{f}}$.

Proof For $\boldsymbol{w} \in \boldsymbol{K}_{\boldsymbol{f}}$, from (3.9) we have

$$
\|\boldsymbol{u}\|_{\boldsymbol{v}} \leq \frac{\alpha_{\boldsymbol{f}}}{2}+\frac{c_{b}}{v-m \lambda_{0}^{-1}} \alpha_{\boldsymbol{f}}^{2} \leq \frac{\alpha_{\boldsymbol{f}}}{2}+\frac{\alpha_{\boldsymbol{f}}}{2}=\alpha_{\boldsymbol{f}} .
$$

Hence, $\boldsymbol{u} \in \boldsymbol{K}_{\boldsymbol{f}}$.

Theorem 3.7 Assume $H(\psi), \boldsymbol{f} \in \boldsymbol{V}^{*}$, and (3.12). Then Problem 2.4 has a unique solution $\boldsymbol{u} \in \boldsymbol{V}$.

Proof Define an operator $\Lambda: \boldsymbol{K}_{\boldsymbol{f}} \rightarrow \boldsymbol{K}_{\boldsymbol{f}}$ by

$$
\Lambda \boldsymbol{w}=\boldsymbol{u}
$$

where $\boldsymbol{u} \in \boldsymbol{K}_{\boldsymbol{f}}$ denotes the unique solution of Problem 3.4. Let us prove that the operator $\Lambda: \boldsymbol{K}_{\boldsymbol{f}} \rightarrow \boldsymbol{K}_{\boldsymbol{f}}$ is a contraction.

Let $\boldsymbol{w}_{1}, \boldsymbol{w}_{2} \in \boldsymbol{K}_{\boldsymbol{f}}$ and $\boldsymbol{u}_{1}=\Lambda \boldsymbol{w}_{1}, \boldsymbol{u}_{2}=\Lambda \boldsymbol{w}_{2}$ be the unique solutions of Problem 3.4 corresponding to $\boldsymbol{w}_{1}, \boldsymbol{w}_{2}$, respectively. Then, for any $\boldsymbol{v} \in \boldsymbol{V}$,

$$
a\left(\boldsymbol{u}_{1}-\boldsymbol{u}_{2}, \boldsymbol{v}\right)+\int_{\Gamma}\left[\psi^{0}\left(u_{1 n} ; v_{n}\right)+\psi^{0}\left(u_{2 n} ;-v_{n}\right)\right] d s \geq b\left(\boldsymbol{w}_{2}, \boldsymbol{w}_{2}, \boldsymbol{v}\right)-b\left(\boldsymbol{w}_{1}, \boldsymbol{w}_{1}, \boldsymbol{v}\right) .
$$

Take $\boldsymbol{v}=\boldsymbol{u}_{2}-\boldsymbol{u}_{1}$ in (3.13), we obtain

$$
\begin{aligned}
v\left\|\boldsymbol{u}_{1}-\boldsymbol{u}_{2}\right\|_{\boldsymbol{V}}^{2}= & a\left(\boldsymbol{u}_{1}-\boldsymbol{u}_{2}, \boldsymbol{u}_{1}-\boldsymbol{u}_{2}\right) \\
\leq & \int_{\Gamma}\left[\psi^{0}\left(u_{1 n} ; u_{2 n}-u_{1 n}\right)+\psi^{0}\left(u_{2 n} ; u_{1 n}-u_{2 n}\right)\right] d s \\
& +b\left(\boldsymbol{w}_{2}, \boldsymbol{w}_{2}, \boldsymbol{u}_{1}-\boldsymbol{u}_{2}\right)-b\left(\boldsymbol{w}_{1}, \boldsymbol{w}_{1}, \mathbf{u}_{1}-\mathbf{u}_{2}\right) .
\end{aligned}
$$

Note that

$$
\int_{\Gamma}\left[\psi^{0}\left(u_{1 n} ; u_{2 n}-u_{1 n}\right)+\psi^{0}\left(u_{2 n} ; u_{1 n}-u_{2 n}\right)\right] d s \leq m \lambda_{0}^{-1}\left\|\boldsymbol{u}_{1}-\boldsymbol{u}_{2}\right\|_{V}^{2}
$$


and

$$
\begin{aligned}
& b\left(\boldsymbol{w}_{2}, \boldsymbol{w}_{2}, \boldsymbol{u}_{1}-\boldsymbol{u}_{2}\right)-b\left(\boldsymbol{w}_{1}, \boldsymbol{w}_{1}, \boldsymbol{u}_{1}-\boldsymbol{u}_{2}\right) \\
& \quad=b\left(\boldsymbol{w}_{2}-\boldsymbol{w}_{1}, \boldsymbol{w}_{2}, \boldsymbol{u}_{1}-\boldsymbol{u}_{2}\right)-b\left(\boldsymbol{w}_{1}, \boldsymbol{w}_{1}-\boldsymbol{w}_{2}, \boldsymbol{u}_{1}-\boldsymbol{u}_{2}\right) \\
& \quad \leq c_{b}\left(\left\|\boldsymbol{w}_{1}\right\| \boldsymbol{v}+\left\|\boldsymbol{w}_{2}\right\| \boldsymbol{v}\right)\left\|\boldsymbol{u}_{1}-\boldsymbol{u}_{2}\right\| \boldsymbol{v}\left\|\boldsymbol{w}_{1}-\boldsymbol{w}_{2}\right\| \boldsymbol{v} .
\end{aligned}
$$

Thus,

$$
\begin{aligned}
\left\|\Lambda \boldsymbol{w}_{1}-\Lambda \boldsymbol{w}_{2}\right\| \boldsymbol{v} & =\left\|\boldsymbol{u}_{1}-\boldsymbol{u}_{2}\right\| \boldsymbol{v} \leq \frac{c_{b}\left(\left\|\boldsymbol{w}_{1}\right\| \boldsymbol{v}+\left\|\boldsymbol{w}_{2}\right\| \boldsymbol{v}\right)}{v-m \lambda_{0}^{-1}}\left\|\boldsymbol{w}_{1}-\boldsymbol{w}_{2}\right\| \boldsymbol{v} \\
& \leq \beta_{\boldsymbol{f}}\left\|\boldsymbol{w}_{1}-\boldsymbol{w}_{2}\right\| \boldsymbol{v},
\end{aligned}
$$

where

$$
\beta_{\boldsymbol{f}}=\frac{2 c_{b} \alpha_{\boldsymbol{f}}}{v-m \lambda_{0}^{-1}} .
$$

By the smallness condition (3.12),

$$
\beta_{\boldsymbol{f}}<1 \text {. }
$$

Hence, the operator $\Lambda: \boldsymbol{K}_{\boldsymbol{f}} \rightarrow \boldsymbol{K}_{\boldsymbol{f}}$ is contractive. Applying the Banach fixed point theorem (cf. [1, Theorem 5.1.3]), we deduce that there exists a unique $\boldsymbol{u}^{*} \in \boldsymbol{K}_{\boldsymbol{f}}$ such that $\Lambda \boldsymbol{u}^{*}=\boldsymbol{u}^{*}$.

By the definition of $\Lambda, \boldsymbol{u}^{*} \in \boldsymbol{K}_{\boldsymbol{f}}$ satisfies

$$
a\left(\boldsymbol{u}^{*}, \boldsymbol{v}\right)+b\left(\boldsymbol{u}^{*}, \boldsymbol{u}^{*}, \boldsymbol{v}\right)+\int_{\Gamma} \psi^{0}\left(u_{n}^{*} ; v_{n}\right) d s \geq\langle\boldsymbol{f}, \boldsymbol{v}\rangle \quad \forall \boldsymbol{v} \in \boldsymbol{V}
$$

That is, $\boldsymbol{u}^{*} \in \boldsymbol{K}_{\boldsymbol{f}}$ is a solution of Problem 2.4.

We now prove the uniqueness of the solution to Problem 2.4. Let $\boldsymbol{u}_{1} \in \boldsymbol{K}_{\boldsymbol{f}}$ and $\boldsymbol{u}_{2} \in \boldsymbol{K}_{\boldsymbol{f}}$ be two solutions to Problem 2.4. Then, for any $\boldsymbol{v} \in \boldsymbol{V}$,

$$
a\left(\boldsymbol{u}_{1}-\boldsymbol{u}_{2}, \boldsymbol{v}\right)+b\left(\boldsymbol{u}_{1}, \boldsymbol{u}_{1}, \boldsymbol{v}\right)-b\left(\boldsymbol{u}_{2}, \boldsymbol{u}_{2}, \boldsymbol{v}\right)+\int_{\Gamma}\left[\psi^{0}\left(u_{1 n} ; v_{n}\right)+\psi^{0}\left(u_{2 n} ;-v_{n}\right)\right] d s \geq 0
$$

Taking $\boldsymbol{v}=\boldsymbol{u}_{2}-\boldsymbol{u}_{1}$ in (3.15), we obtain

$$
\begin{aligned}
v\left\|\boldsymbol{u}_{1}-\boldsymbol{u}_{2}\right\|_{\boldsymbol{v}}^{2}= & a\left(\boldsymbol{u}_{1}-\boldsymbol{u}_{2}, \boldsymbol{u}_{1}-\boldsymbol{u}_{2}\right) \\
\leq & \int_{\Gamma}\left[\psi^{0}\left(u_{1 n} ; u_{2 n}-u_{1 n}\right)+\psi^{0}\left(u_{2 n} ; u_{1 n}-u_{2 n}\right)\right] d s \\
& +b\left(\boldsymbol{u}_{2}, \boldsymbol{u}_{2}, \boldsymbol{u}_{1}-\boldsymbol{u}_{2}\right)-b\left(\boldsymbol{u}_{1}, \boldsymbol{u}_{1}, \boldsymbol{u}_{1}-\boldsymbol{u}_{2}\right) \\
\leq & m \lambda_{0}^{-1}\left\|\boldsymbol{u}_{1}-\boldsymbol{u}_{2}\right\|_{\boldsymbol{V}}^{2}+b\left(\boldsymbol{u}_{2}-\boldsymbol{u}_{1}, \boldsymbol{u}_{2}, \boldsymbol{u}_{1}-\boldsymbol{u}_{2}\right) \\
\leq & m \lambda_{0}^{-1}\left\|\boldsymbol{u}_{1}-\boldsymbol{u}_{2}\right\|_{\boldsymbol{V}}^{2}+c_{b}\left\|\boldsymbol{u}_{2}\right\| \boldsymbol{v}\left\|\boldsymbol{u}_{1}-\boldsymbol{u}_{2}\right\|_{\boldsymbol{V}}^{2} .
\end{aligned}
$$

Hence,

$$
\left\|\boldsymbol{u}_{1}-\boldsymbol{u}_{2}\right\|_{\boldsymbol{v}}^{2} \leq \frac{\beta_{\boldsymbol{f}}}{2}\left\|\boldsymbol{u}_{1}-\boldsymbol{u}_{2}\right\|_{\boldsymbol{v}}^{2}
$$


Since $\beta_{\boldsymbol{f}}<1$, we deduce from the above inequality that $\boldsymbol{u}_{1}=\boldsymbol{u}_{2}$. This completes the proof of the theorem.

\subsection{A convergent iteration algorithm}

In addition to the existence of a unique fixed point for a contractive mapping over a closed set of a complete space, the Banach fixed point theorem also provides the convergence of a fixed point iteration algorithm. Consider the following iteration algorithm.

Algorithm 3.8 Initialization: Choose an initial guess $\boldsymbol{u}_{0} \in \boldsymbol{K}_{\boldsymbol{f}}$, e.g., $\boldsymbol{u}_{0}=\mathbf{0}$.

Iteration: For $k \geq 1$, find $\boldsymbol{u}_{k} \in \boldsymbol{V}$ such that

$$
a\left(\boldsymbol{u}_{k}, \boldsymbol{v}\right)+\int_{\Gamma} \psi^{0}\left(u_{k, n} ; v_{n}\right) d s \geq\langle\boldsymbol{f}, \boldsymbol{v}\rangle-b\left(\boldsymbol{u}_{k-1}, \boldsymbol{u}_{k-1}, \boldsymbol{v}\right) \quad \forall \boldsymbol{v} \in \boldsymbol{V} .
$$

As an application of [1, Theorem 5.1.3], we have the following result.

Theorem 3.9 Assume $H(\psi), \boldsymbol{f} \in \boldsymbol{V}^{*}$ and (3.12). Let $\mathbf{u} \in \boldsymbol{V}$ be the unique solution of Problem 2.4. Then the sequence $\left\{\boldsymbol{u}_{k}\right\} \subset \boldsymbol{K}_{\boldsymbol{f}}$ defined by (3.16) converges to $\boldsymbol{u}$ :

$$
\left\|\boldsymbol{u}_{k}-\boldsymbol{u}\right\|_{\boldsymbol{v}} \rightarrow 0 \quad \text { as } k \rightarrow \infty
$$

The following error estimates are valid:

$$
\begin{aligned}
\left\|\boldsymbol{u}_{k}-\boldsymbol{u}\right\|_{\boldsymbol{v}} & \leq \beta_{\boldsymbol{f}}\left\|\boldsymbol{u}_{k-1}-\boldsymbol{u}\right\|_{\boldsymbol{v}}, \\
\left\|\boldsymbol{u}_{k}-\boldsymbol{u}\right\|_{\boldsymbol{v}} & \leq \frac{\beta_{\boldsymbol{f}}}{1-\beta_{\boldsymbol{f}}}\left\|\boldsymbol{u}_{k-1}-\boldsymbol{u}_{k}\right\|_{\boldsymbol{v}}, \\
\left\|\boldsymbol{u}_{k}-\boldsymbol{u}\right\|_{\boldsymbol{v}} & \leq \frac{\beta_{\boldsymbol{f}}^{k}}{1-\beta_{\boldsymbol{f}}}\left\|\boldsymbol{u}_{0}-\boldsymbol{u}_{1}\right\|_{\boldsymbol{v}} .
\end{aligned}
$$

Remark 3.10 Under the smallness condition (3.12), the constant $\beta_{\boldsymbol{f}}$ defined by (3.14) satisfies $\beta_{\boldsymbol{f}}<1$. The first error estimate (3.17) shows that the iteration algorithm converges linearly with the convergence rate $\beta_{\boldsymbol{f}}$. The second bound (3.18) is an a-posteriori error estimate: once the iterates $\boldsymbol{u}_{k-1}$ and $\boldsymbol{u}_{k}$ are available, they can be used to provide a computable upper bound of the error $\left\|\boldsymbol{u}_{k}-\boldsymbol{u}\right\|_{\boldsymbol{v}}$. The third bound (3.19) is an a-priori error estimate: once $\boldsymbol{u}_{0}$ is chosen and $\boldsymbol{u}_{1}$ is calculated, it allows one to find the number of iterations needed to guarantee the error $\left\|\boldsymbol{u}_{k}-\boldsymbol{u}\right\|_{\boldsymbol{v}}$ to be in a given range.

Problem (3.16) is a Stokes-type hemivariational inequality in which the differential operator for the unknown variable $\boldsymbol{u}_{k}$ is linear. Obviously, it is easier to solve (3.16) than Problem 2.4 in which the differential operator for the unknown variable $\boldsymbol{u}_{k}$ is nonlinear. When Problem 2.4 is solved by a numerical method, e.g., the finite element, the discretized Navier-Stokes hemivariational inequality can be similarly approximated by Algorithm 3.8 at the discrete level. Under the stated assumptions, we again have convergence and the three error estimates, all for the discrete solutions.

\subsection{Lipschitz continuous dependence on the right-hand side}

Finally, we provide a Lipschitz continuous dependence result for the solution $\boldsymbol{u}$ of Problem 2.4 on the right-hand side $\boldsymbol{f}$. 
For $m_{0}>0$, define a subset $\boldsymbol{V}_{m_{0}}^{*} \subset \boldsymbol{V}^{*}$ by

$$
\boldsymbol{V}_{m_{0}}^{*}=\left\{\boldsymbol{f} \in \boldsymbol{V}^{*}:\|\boldsymbol{f}\|_{\boldsymbol{V}^{*}} \leq m_{0}\right\}
$$

To derive the Lipschitz continuous dependence result, we assume $\boldsymbol{f} \in \boldsymbol{V}_{m_{0}}^{*}$ from now on. Similar to (3.11), define

$$
\alpha_{m_{0}}=\frac{2\left(c_{0} \lambda_{0}^{-1 / 2}|\Gamma|^{1 / 2}+m_{0}\right)}{v-m \lambda_{0}^{-1}} .
$$

Theorem 3.11 Assume $H(\psi), \boldsymbol{f} \in \boldsymbol{V}_{m_{0}}^{*}$, and

$$
0<2 c_{b} \alpha_{m_{0}}<v-m \lambda_{0}^{-1}
$$

Then the solution $\boldsymbol{u}$ of Problem 2.4 depends Lipschitz continuously on $\boldsymbol{f}$.

Proof Let $\boldsymbol{f}_{1}, \boldsymbol{f}_{2} \in \boldsymbol{V}_{m_{0}}^{*}$ and $\boldsymbol{u}_{1}, \boldsymbol{u}_{2} \in \boldsymbol{V}$ be the unique solutions of Problem 2.4 corresponding to $\boldsymbol{f}_{1}, \boldsymbol{f}_{2}$, respectively. Then, for any $\boldsymbol{v} \in \boldsymbol{V}$,

$$
\begin{aligned}
& a\left(\boldsymbol{u}_{1}-\boldsymbol{u}_{2}, \boldsymbol{v}\right)+b\left(\boldsymbol{u}_{1}, \boldsymbol{u}_{1}, \boldsymbol{v}\right)-b\left(\boldsymbol{u}_{2}, \boldsymbol{u}_{2}, \boldsymbol{v}\right) \\
& \quad+\int_{\Gamma}\left[\psi^{0}\left(u_{1 n} ; v_{n}\right)+\psi^{0}\left(u_{2 n} ;-v_{n}\right)\right] d s \geq\left\langle\boldsymbol{f}_{1}-\boldsymbol{f}_{2}, \boldsymbol{v}\right\rangle .
\end{aligned}
$$

Take $\boldsymbol{v}=\boldsymbol{u}_{2}-\boldsymbol{u}_{1}$ in (3.22), we obtain

$$
\begin{aligned}
v\left\|\boldsymbol{u}_{1}-\boldsymbol{u}_{2}\right\|_{\boldsymbol{v}}^{2}= & a\left(\boldsymbol{u}_{1}-\boldsymbol{u}_{2}, \boldsymbol{u}_{1}-\boldsymbol{u}_{2}\right) \\
\leq & \int_{\Gamma}\left[\psi^{0}\left(u_{1 n} ; u_{2 n}-u_{1 n}\right)+\psi^{0}\left(u_{2 n} ; u_{1 n}-u_{2 n}\right)\right] d s \\
& +b\left(\boldsymbol{u}_{2}, \boldsymbol{u}_{2}, \boldsymbol{u}_{1}-\boldsymbol{u}_{2}\right)-b\left(\boldsymbol{u}_{1}, \boldsymbol{u}_{1}, \boldsymbol{u}_{1}-\boldsymbol{u}_{2}\right)+\left\langle\boldsymbol{f}_{1}-\boldsymbol{f}_{2}, \boldsymbol{u}_{1}-\boldsymbol{u}_{2}\right\rangle \\
\leq & m \lambda_{0}^{-1}\left\|\boldsymbol{u}_{1}-\boldsymbol{u}_{2}\right\|_{\boldsymbol{V}}^{2}+b\left(\boldsymbol{u}_{2}-\boldsymbol{u}_{1}, \boldsymbol{u}_{2}, \boldsymbol{u}_{1}-\boldsymbol{u}_{2}\right)+\left\|\boldsymbol{f}_{1}-\boldsymbol{f}_{2}\right\| \boldsymbol{v}^{*}\left\|\boldsymbol{u}_{1}-\boldsymbol{u}_{2}\right\|_{\boldsymbol{v}} \\
\leq & m \lambda_{0}^{-1}\left\|\boldsymbol{u}_{1}-\boldsymbol{u}_{2}\right\|_{\boldsymbol{V}}^{2}+c_{b}\left\|\boldsymbol{u}_{2}\right\| \boldsymbol{v}\left\|\boldsymbol{u}_{1}-\boldsymbol{u}_{2}\right\|_{\boldsymbol{v}}^{2}+\left\|\boldsymbol{f}_{1}-\boldsymbol{f}_{2}\right\| \boldsymbol{v}^{*}\left\|\boldsymbol{u}_{1}-\boldsymbol{u}_{2}\right\| \boldsymbol{v} .
\end{aligned}
$$

From Theorem 3.7,

$$
\left\|\boldsymbol{u}_{2}\right\| \boldsymbol{v} \leq \alpha_{m_{0}}
$$

Applying condition (3.21), we derive the inequality

$$
\left\|\boldsymbol{u}_{1}-\boldsymbol{u}_{2}\right\| \boldsymbol{v} \leq \frac{2}{v-m \lambda_{0}^{-1}}\left\|\boldsymbol{f}_{1}-\boldsymbol{f}_{2}\right\| \boldsymbol{v}^{*},
$$

which shows the Lipschitz continuity of the solution $\boldsymbol{u}$ with respect to $\boldsymbol{f}$.

\section{Conclusions}

We analyze a stationary Navier-Stokes HVI through convex minimization and Banach fixed-point argument. In the existing literature, the solution existence of the Navier- 
Stokes HVI is proved through an application of an abstract surjectivity result for pseudomonotone operators. In this paper, we establish a minimization principle for an auxiliary Stokes HVI, and prove the solution existence and uniqueness of the Navier-Stokes HVI by applying the Banach fixed point theorem. This treatment naturally leads to a convergent iteration algorithm, where the unique solution of the stationary Navier-Stokes HVI can be obtained as the limit of a sequence of solutions to the Stokes HVIs.

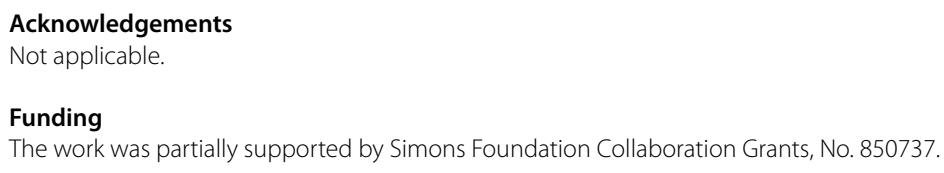

\section{Declarations}

Competing interests

The authors declare that they have no competing interests.

\section{Authors' contributions}

All authors contributed equally to this paper. All authors read and approved the final manuscript.

\section{Author details}

'School of Mathematics and Statistics, Xi'an Jiaotong University, Xi'an, Shaanxi 710049, China. ${ }^{2}$ Department of Mathematics, University of lowa, lowa City, IA 52242-1410, USA.

\section{Publisher's Note}

Springer Nature remains neutral with regard to jurisdictional claims in published maps and institutional affiliations.

Received: 19 August 2021 Accepted: 11 October 2021 Published online: 01 December 2021

\section{References}

1. Atkinson, K., Han, W.: Theoretical Numerical Analysis: A Functional Analysis Framework, 3rd edn. Springer, New York (2009)

2. Clarke, F.H.: Optimization and Nonsmooth Analysis. Wiley-Interscience, New York (1983)

3. Fan, L., Liu, S., Gao, S.: Generalized monotonicity and convexity of non-differentiable functions. J. Math. Anal. Appl. $279,276-289(2003)$

4. Fang, C., Czuprynski, K., Han, W., Cheng, X.L., Dai, X.: Finite element method for a stationary Stokes hemivariational inequality with slip boundary condition. IMA J. Numer. Anal. 40, 2696-2716 (2020)

5. Fang, C., Han, W.: Well-posedness and optimal control of a hemivariational inequality for nonstationary Stokes fluid flow. Discrete Contin. Dyn. Syst. 36, 5369-5386 (2016)

6. Fang, C., Han, W., Migórski, S., Sofonea, M.: A class of hemivariational inequalities for nonstationary Navier-Stokes equations. Nonlinear Anal., Real World Appl. 31, 257-276 (2016)

7. Girault, V., Raviart, P.A.: Finite Element Methods for Navier-Stokes Equations: Theory and Algorithms. Springer, Berlin (1986)

8. Han, W.: Minimization principles for elliptic hemivariational inequalities. Nonlinear Anal., Real World Appl. 54, article number $103114(2020)$

9. Han, W.: A revisit of elliptic variational-hemivariational inequalities. Numer. Funct. Anal. Optim. 42, 371-395 (2021)

10. Han, W., Czuprynski, K., Jing, F.: Mixed finite element method for a hemivariational inequality of stationary Navier-Stokes equations. J. Sci. Comput. 89, article number 8 (2021)

11. Han, W., Sofonea, M.: Numerical analysis of hemivariational inequalities in contact mechanics. Acta Numer. 28 , 175-286 (2019)

12. Haslinger, J., Miettinen, M., Panagiotopoulos, P.D.: Finite Element Method for Hemivariational Inequalities: Theory, Methods and Applications. Kluwer Academic, Boston (1999)

13. Lamb, H.: On the conditions for steady motion of a fluid. Proc. Lond. Math. Soc. s1-9(1), 91-93 (1877)

14. Ling, M., Han, W.: Minimization principle in study of a Stokes hemivariational inequality. Appl. Math. Lett. 121, article number 107401 (2021)

15. Ling, M., Wang, F., Han, W.: The nonconforming virtual element method for a stationary Stokes hemivariational inequality with slip boundary condition. J. Sci. Comput. 85, article number 56 (2020)

16. Migórski, S.: Hemivariational inequalities modeling viscous incompressible fluids. J. Nonlinear Convex Anal. $\mathbf{5}$, 217-227 (2004)

17. Migórski, S., Ochal, A.: Hemivariational inequalities for stationary Navier-Stokes equations. J. Math. Anal. Appl. 306 197-217 (2005) 
18. Migórski, S., Ochal, A.: Navier-Stokes problems modeled by evolution hemivariational inequalities. Discrete Contin. Dyn. Syst. 2007, 731-740 (2007)

19. Migórski, S., Ochal, A., Sofonea, M.: Nonlinear Inclusions and Hemivariational Inequalities: Models and Analysis of Contact Problems. Springer, New York (2013)

20. Naniewicz, Z., Panagiotopoulos, P.D.: Mathematical Theory of Hemivariational Inequalities and Applications. Dekker, New York (1995)

21. Panagiotopoulos, P.D.: Hemivariational Inequalities, Applications in Mechanics and Engineering. Springer, Berlin (1993)

22. Sofonea, M., Migórski, S.: Variational-Hemivariational Inequalities with Applications. Pure and Applied Mathematics. Chapman \& Hall, Boca Raton (2018)

Submit your manuscript to a SpringerOpen ${ }^{\circ}$ journal and benefit from:

- Convenient online submission

Rigorous peer review

- Open access: articles freely available online

- High visibility within the field

- Retaining the copyright to your article

Submit your next manuscript at $\boldsymbol{\nabla}$ springeropen.com 